(n)

trobertivier Journal of Nonlinear Mathematical Physics

\title{
On Decomposition of the ABS Lattice Equations and Related
}

\section{Bäcklund Transformations}

Danda Zhang, Da-jun Zhang

To cite this article: Danda Zhang, Da-jun Zhang (2018) On Decomposition of the ABS Lattice Equations and Related Bäcklund Transformations, Journal of Nonlinear Mathematical Physics 25:1, 34-53, DOI:

https://doi.org/10.1080/14029251.2018.1440741

To link to this article: https://doi.org/10.1080/14029251.2018.1440741

Published online: 04 January 2021 


\title{
On Decomposition of the ABS Lattice Equations and Related Bäcklund Transformations
}

\author{
Danda Zhang, Da-jun Zhang * \\ Department of Mathematics, Shanghai University \\ Shanghai, 200444, People's Republic of China \\ djzhang@staff.shu.edu.cn
}

Received 10 May 2017

Accepted 7 August 2017

\begin{abstract}
The Adler-Bobenko-Suris (ABS) list contains scalar quadrilateral equations which are consistent around the cube, and have $D_{4}$ symmetry and tetrahedron property. Each equation in the ABS list admits a beautiful decomposition. We revisit these decomposition formulas and by means of them we construct Bäcklund transformations (BTs). BTs are used to construct lattice equations, their new solutions and weak Lax pairs.
\end{abstract}

Keywords: ABS list; decomposition; Bäcklund transformation; solutions; weak Lax pair.

2000 Mathematics Subject Classification: 35Q51, 35Q55, 37K05, 39A99

\section{Introduction}

It is well known that discrete integrable systems play important roles in variety of areas such as statistic physics, discrete differential geometry and discrete Painlevé theory. Quadrilateral equations are partial difference equations defined on four points (see Fig.1(a)), with a form

$$
Q(u, \widetilde{u}, \widehat{u}, \widehat{\widetilde{u}} ; p, q)=0,
$$

where $u$ is a function of discrete variables $n, m$, constants $p, q$ serve as spacing parameters of $n$ direction and $m$-direction, respectively. Short hand notations

$$
u=u_{n, m}, \quad \widetilde{u}=u_{n+1, m}, \quad \widehat{u}=u_{n, m+1}, \widehat{\widetilde{u}}=u_{n+1, m+1}
$$

will be used in the paper. When we speak of consistency-around-the-cube property of the quadrilateral equation (1.1), we mean that [10,21]: (i) equation (1.1) is affine linear, (ii) imposing on the six faces of the cube (see Fig.1(b)) six equations of type (1.1),

$$
\begin{aligned}
& Q(u, \widetilde{u}, \widehat{u}, \widehat{\widetilde{u}} ; p, q)=0, Q(\bar{u}, \overline{\widetilde{u}}, \overline{\widehat{u}}, \overline{\widetilde{\widetilde{u}}} ; p, q)=0, \\
& Q(u, \widetilde{u}, \bar{u}, \widetilde{\bar{u}} ; p, r)=0, Q(\widehat{u}, \widehat{\widetilde{u}}, \widehat{\bar{u}}, \widehat{\bar{u}} ; p, r)=0, \\
& Q(u, \widehat{u}, \bar{u}, \widehat{\bar{u}} ; q, r)=0, Q(\widetilde{u}, \widetilde{\widehat{u}}, \widetilde{\bar{u}}, \widetilde{\bar{u}} ; q, r)=0,
\end{aligned}
$$

where shift in the third direction is denoted by bar and $r$ is spacing parameter of the third direction, one first solves the doubly shifted quantities from the left hand side of (1.2) and then the triply

${ }^{*}$ Corresponding author. 
(a)

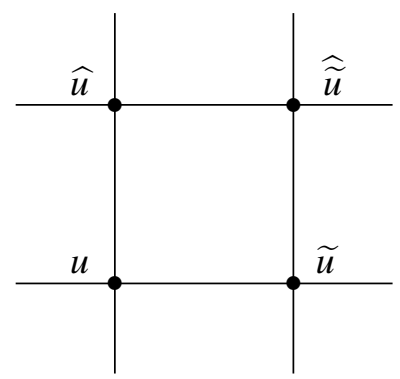

(b)

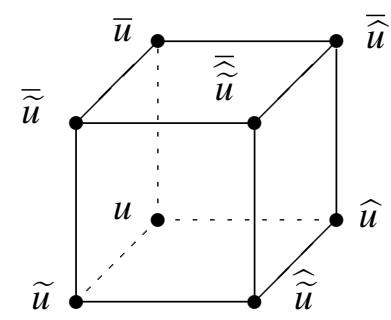

Fig. 1. (a). An elementary quadrilateral. (b). The consistency cube.

shifted quantity obtained from the right hand side of (1.2) are the same. In a beautiful paper [1] Adler Bobenko and Suris (ABS) classified all quadrilateral equations with the assumption of consistencyaround-the-cube and additional conditions: (i) equation (1.1) having $D_{4}$ symmetry; (ii) tetrahedron property, i.e. the final value of $\widehat{\widetilde{u}}$ only depends on $\widetilde{u}, \widehat{u}, \bar{u}$. ABS list includes all such consistentaround-the-cube (CAC) equations:

$$
\begin{aligned}
& \mathrm{H} 1: \quad(u-\widehat{\widetilde{u}})(\widetilde{u}-\widehat{u})-p+q=0, \\
& \mathrm{H} 2:(u-\widehat{\widetilde{u}})(\widetilde{u}-\widehat{u})-(p-q)(u+\widetilde{u}+\widehat{u}+\widehat{\widetilde{u}}+p+q)=0, \\
& \mathrm{H} 3(\delta): p(u \tilde{u}+\widehat{u} \widehat{\bar{u}})-q(u \widehat{u}+\widehat{\widetilde{u}})+\delta\left(p^{2}-q^{2}\right)=0, \\
& \operatorname{A} 1(\delta): p(u+\widehat{u})(\widetilde{u}+\widehat{\widetilde{u}})-q(u+\widetilde{u})(\widehat{u}+\widehat{\widetilde{u}})-\delta^{2} p q(p-q)=0, \\
& \text { A2: } p\left(1-q^{2}\right)(u \widetilde{u}+\widehat{u} \widehat{\vec{u}})-q\left(1-p^{2}\right)(u \widehat{u}+\widetilde{u} \widehat{u}) \\
& -\left(p^{2}-q^{2}\right)(1+u \widetilde{u} \hat{u} \widehat{\bar{u}})=0, \\
& \mathrm{Q} 1(\delta): p(u-\widehat{u})(\widetilde{u}-\widehat{\widetilde{u}})-q(u-\widetilde{u})(\widehat{u}-\widehat{\widetilde{u}})+\delta^{2} p q(p-q)=0 \text {, } \\
& \text { Q2: } p(u-\widehat{u})(\widetilde{u}-\widehat{\widetilde{u}})-q(u-\widetilde{u})(\widehat{u}-\widehat{\widetilde{u}}) \\
& +p q(p-q)\left(u+\widetilde{u}+\widehat{u}+\widehat{\widetilde{u}}-p^{2}+p q-q^{2}\right)=0, \\
& \mathrm{Q} 3(\delta): p\left(1-q^{2}\right)(u \widehat{u}+\widetilde{u} \widehat{u})-q\left(1-p^{2}\right)(u \widetilde{u}+\widehat{u} \widehat{\bar{u}}) \\
& -\left(p^{2}-q^{2}\right)\left(\widetilde{u} \widehat{u}+u \widehat{\widetilde{u}}+\frac{\delta^{2}\left(1-p^{2}\right)\left(1-q^{2}\right)}{4 p q}\right)=0, \\
& \text { Q4: } \operatorname{sn}(p)(u \widetilde{u}+\widehat{u} \widehat{\vec{u}})-\operatorname{sn}(q)(u \widehat{u}+\widehat{\widetilde{u}})-\operatorname{sn}(p-q)(\widetilde{u} \widehat{u}+u \widehat{\widetilde{u}}) \\
& +\operatorname{sn}(p) \operatorname{sn}(q) \operatorname{sn}(p-q)\left(1+k^{2} u \widetilde{u} \widehat{u} \bar{u}\right)=0,
\end{aligned}
$$

where $\delta$ is an arbitrary parameter, $\operatorname{sn}(p)=\operatorname{sn}(p ; k)$ is the Jacobi elliptic function and $Q 4$ in the above form was given by Hietarinta [9].

If the top equation in the consistency cube is viewed as same as the bottom equation but with $\bar{u}$ as a new solution, then the side equations as a coupled system, i.e.

$$
Q(u, \widetilde{u}, \bar{u}, \widetilde{\bar{u}} ; p, r)=0, Q(u, \widehat{u}, \bar{u}, \widehat{\bar{u}} ; q, r)=0,
$$

automatically provides a Bäcklund transformation (BT) for the bottom equation (1.1). Its linearized form obtained by introducing $\bar{u}=g / f$ can act as a Lax pair of equation (1.1). The equations on the bottom and top faces have the same form at the beginning, but if one imposes different limits 
on the top and bottom variables then the resulting equations will be different and the two side equations will give a BT of the two resulting equations. This beautiful idea was demonstrated in [3] by Atkinson. Usually by an auto-BT we mean it connects different solutions of same equation while by a nonauto-BT we mean it connects solutions of two different equations.

BT originated from the construction of pseudo-spherical surfaces and BTs have been playing important roles in soliton theory [15,22,23]. In this paper, we will consider the ABS list and focus on those BTs that can be constructed by using decomposition property of the ABS equations. In fact, each equation $Q=0$ in the $\mathrm{ABS}$ list admits a decomposition which is an analogue of the following,

$$
\mathscr{H}=h^{12} h^{34}-h^{13} h^{24}=P Q, P=\left|\begin{array}{ccc}
Q & Q_{u_{1}} & Q_{u_{4}} \\
Q_{u_{2}} & Q_{u_{1} u_{2}} & Q_{u_{2} u_{4}} \\
Q_{u_{3}} & Q_{u_{1} u_{3}} & Q_{u_{3} u_{4}}
\end{array}\right|,
$$

where $u_{1}=u, u_{2}=\widetilde{u}, u_{3}=\widehat{u}, u_{4}=\widehat{\widetilde{u}}$,

$$
h^{i j}\left(u_{i}, u_{j}\right)=Q_{u_{k}} Q_{u_{l}}-Q Q_{u_{k} u_{l}},
$$

$Q_{u_{k}}=\partial_{u_{k}} Q, Q_{u_{i} u_{k}}=\partial_{u_{i}} \partial_{u_{k}} Q$, and $i, j, k, l$ are distinct elements in $\{1,2,3,4\}$. The decomposition (1.5) played a crucial role in the classification of the ABS list and in the further discussions under the CAC property with a more general setting (allowing the faces of the consistency cube to carry a priori different quad-equations) $[2,7,8] . h^{i j}$ are used to study singularity structures of solutions, which is considered to be related to the CAC property and boundary value structures (cf. $[4,5])$. The decomposition (1.5) and $h$ polynomials can be used to construct BTs. For example, H3 $(\delta)$ has the following decomposition:

$$
\mathscr{H}=h(u, \widetilde{u}, p) h(\widehat{u}, \widehat{\widetilde{u}}, p)-h(u, \widehat{u}, q) h(\widetilde{u}, \widehat{\widetilde{u}}, q)=H 3(\delta)=0
$$

where $h$ is defined by $h(u, \widetilde{u}, p)=u \widetilde{u}+p \delta$. This implies that with unknown function $U$, the pair

$$
h(u, \widetilde{u}, p)=U \widetilde{U}, h(u, \widehat{u}, q)=U \widehat{U}
$$

will provide a BT to connect $\mathrm{H} 3(\delta)$ and the $U$-equation which comes from compatibility of the pair in terms of $u$. By calculation one can find the corresponding $U$-equation is $\mathrm{H} 3(-\delta)$. In this paper we will examine system (1.7) for some ABS equations. As a result, when $h$ is affine linear, a completed list of BTs together with connected CAC equations are given. When $h$ is beyond affine linear, some quadratic CAC equations are obtained. Some BTs are used to construct new solutions and weak Lax pairs.

The paper is organized as follows. In Sec. 2 we revisit decomposition of the ABS list. In Sec.3 we discuss possible forms of $h$ and the related quadrilateral equations of $u$ and $U$, which are listed in Table 1 and 2. Sec.4 includes some examples as applications, a new weak Lax pair of Q1(0), new polynomial solutions of $\mathrm{Q} 1(\delta)$ and rational solutions of $\mathrm{H} 3 *(\delta)$ in Casoratian form are obtained. Finally, Sec.5 is for conclusions. 


\section{Decomposition (1.5) of the ABS list}

\subsection{Decomposition (1.5): revisited}

Let us revisit the decomposition (1.5) and have a look at the relation of $Q$ and $P$ from a general viewpoint. Consider $Q(u, \widetilde{u}, \widehat{u}, \widehat{\widetilde{u}} ; p, q)$ to be a general quadrilateral affine linear polynomial:

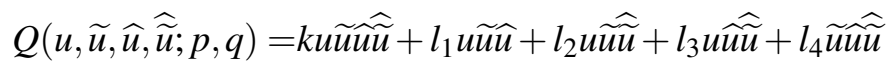

$$
\begin{aligned}
& +p_{1} u \widetilde{u}+p_{2} \widetilde{u} \widehat{u}+p_{3} \widehat{\hat{u}} \widehat{\vec{u}}+p_{4} u \widehat{\widetilde{u}}+p_{5} u \widehat{u}+p_{6} \widehat{\widetilde{u}} \\
& +q_{1} u+q_{2} \widetilde{u}+q_{3} \widehat{u}+q_{4} \widehat{\widetilde{u}}+c,
\end{aligned}
$$

where $k, l_{i}, p_{i}, q_{i}, c$ are constants. Let $\mathscr{P}_{s}^{t}$ denote a set of polynomials with $s$ distinct variables in $\{u, \widetilde{u}, \widehat{u}, \widehat{\widetilde{u}}\}$ and at most degree $t$ for each variable. With this definition, the most general element in $\mathscr{P}_{4}^{1}$ is $Q$ defined in (2.1) and $h^{i j}$ belongs to $\mathscr{P}_{2}^{2}$. For the above generic $Q$, the decomposition (1.5) holds and $P \in \mathscr{P}_{4}^{1}$ [2]. In fact, the discriminant of $h^{i j}$ plays an important role in the classification of integrable quadrilateral equations. With a more general setting in the CAC condition, the classification of $Q$-type equations was done in [2] and a full classification was finished in [7,8].

In the following let us take a close look at the relation between $Q$ and $P$ in (1.5). Similar to (1.6) we define

$$
g^{i j}\left(u_{i}, u_{j}\right)=P_{u_{k}} P_{u_{l}}-P P_{u_{k} u_{l}},
$$

where $P$ is defined in (1.5). Then we have the following.

Proposition 2.1. For the polynomial $Q$ given in (2.1), $P$ defined in (1.5) and $h^{i j}$ in (1.6), there is a constant $K$ such that

$$
g^{i j}=(-1)^{j-i} K h^{i j}, \quad(i, j) \in\{(1,2),(1,3),(2,4),(3,4)\} .
$$

In particular when $K=0, P$ can be factorized as a product of distinct linear function $a_{i} u_{i}+b_{i}$.

Proof. It has been proved that $P \in \mathscr{P}_{4}^{1}$ [2]. Thus we can switch the roles of $Q$ and $P$ in (1.5). For the polynomial $Q$ given in (2.1), $P$ defined in (1.5) and $h^{i j}$ in (1.6), by direct calculation we find

$$
g^{i j}=(-1)^{j-i} K h^{i j}, \quad(i, j) \in\{(1,2),(1,3),(2,4),(3,4)\},
$$

with a same $K$ which is an irreducible rational function of $\left\{u_{i}\right\}$. This implies

$$
g^{12} g^{34}-g^{13} g^{24}=K^{2} Q P .
$$

Corresponding to the structure in (1.5), there must be

$$
K^{2} Q=\left|\begin{array}{ccc}
P & P_{u_{1}} & P_{u_{4}} \\
P_{u_{2}} & P_{u_{1} u_{2}} & P_{u_{2} u_{4}} \\
P_{u_{3}} & P_{u_{1} u_{3}} & P_{u_{3} u_{4}}
\end{array}\right| \in \mathscr{P}_{4}^{1} .
$$

Since $Q \in \mathscr{P}_{4}^{1}$ the only choice for $K$ is a constant. 
If $K=0$, then we have $g^{i j}=0$ in light of (2.3). Noticing that

$$
(\ln P)_{u_{k} u_{l}}=\frac{g^{i j}}{P^{2}}
$$

if $g^{i j}=0$ we have

$$
\ln P=\psi_{1}\left(u_{1}\right)+\psi_{2}\left(u_{2}\right)+\psi_{3}\left(u_{3}\right)+\psi_{4}\left(u_{4}\right),
$$

where $\psi_{i}\left(u_{i}\right)$ is a function of $u_{i}$. This means $P \in \mathscr{P}_{4}^{1}$ can be factorized as

$$
P=\phi_{1}\left(u_{1}\right) \phi_{2}\left(u_{2}\right) \phi_{3}\left(u_{3}\right) \phi_{4}\left(u_{4}\right),
$$

where there must be $\phi_{i}\left(u_{i}\right)=a_{i} u_{i}+b_{i}$ because of $P \in \mathscr{P}_{4}^{1}$.

The above proposition reveals an "adjoint" relation between $Q$ and $P$ if $Q$ is an affine-linear quadrilateral polynomial (2.1). For A-type and Q-type ABS equations, one can see that $Q$ and $P$ are almost same.

\subsection{Decomposition of the ABS list}

For each equation (1.1) in the ABS list it holds that [1]

$$
\mathscr{H}=h(u, \widetilde{u}, p) h(\widehat{u}, \widehat{\widetilde{u}}, p)-h(u, \widehat{u}, q) h(\widetilde{u}, \widehat{\widetilde{u}}, q)=0,
$$

where function $h(u, \tilde{u}, p)$ is $h^{12}$ divided by certain factor $\kappa(p, q)$. Functions $h, \mathscr{H}$ and connections with the ABS equations are listed below:

$\mathrm{H} 1: h(u, \widetilde{u}, p)=1, \mathscr{H}=0$, identity,

$\mathrm{H} 2: h(u, \widetilde{u}, p)=u+\widetilde{u}+p, \quad \mathscr{H}=H 2(u, \widetilde{u}, \widehat{u}, \widehat{\widetilde{u}} ; p, q)=0$,

$\mathrm{H} 3: h(u, \widetilde{u}, p)=u \widetilde{u}+p \delta, \mathscr{H}=H 3(u, \widetilde{u}, \widehat{u}, \widehat{\widetilde{u}} ; p, q)=0$,

$\mathrm{A} 1: h(u, \widetilde{u}, p)=\frac{1}{p}(\widetilde{u}+u)^{2}-\delta^{2} p, \mathscr{H}=A 1(u, \widetilde{u}, \widehat{u}, \widehat{\widetilde{u}} ; p, q) A 1(u, \widetilde{u}, \widehat{u}, \widehat{\widetilde{u}} ; p,-q)=0$,

$\mathrm{A} 2: h(u, \widetilde{u}, p)=\frac{(p u \widetilde{u}-1)(u \widetilde{u}-p)}{1-p^{2}}, \mathscr{H}=A 2(u, \widetilde{u}, \widehat{u}, \widehat{\widetilde{u}} ; p, q) A 2\left(u, \widetilde{u}, \widehat{u}, \widehat{\widetilde{u}} ; p, q^{-1}\right)=0$,

$\mathrm{Q} 1: h(u, \widetilde{u}, p)=\frac{1}{p}(\widetilde{u}-u)^{2}-\delta^{2} p, \quad \mathscr{H}=Q 1(u, \widetilde{u}, \widehat{u}, \widehat{\widetilde{u}} ; p, q) Q 1(u, \widetilde{u}, \widehat{u}, \widehat{\widetilde{u}} ; p,-q)=0$,

$\mathrm{Q} 2: h(u, \widetilde{u}, p)=\frac{1}{p}(\widetilde{u}-u)^{2}-2 p(u+\widetilde{u})+p^{3}$,

$$
\mathscr{H}=Q 2(u, \widetilde{u}, \widehat{u}, \widehat{\widetilde{u}} ; p, q) Q 2(u, \widetilde{u}, \widehat{u}, \widehat{\widetilde{u}} ; p,-q)=0,
$$

Q3 : $h(u, \widetilde{u}, p)=\frac{p}{1-p^{2}}\left(u^{2}+\widetilde{u}^{2}\right)-\frac{1+p^{2}}{1-p^{2}} u \widetilde{u}+\frac{\left(1-p^{2}\right) \delta^{2}}{4 p}$,

$$
\mathscr{H}=Q 3(u, \widetilde{u}, \widehat{u}, \widehat{\widetilde{u}} ; p, q) Q 3\left(u, \widetilde{u}, \widehat{u}, \widehat{\widetilde{u}} ; p, q^{-1}\right)=0,
$$

Q4 : $h(u, \widetilde{u}, p)=\frac{-1}{\operatorname{sn}(p)}\left(k^{2} \operatorname{sn}^{2}(p) u^{2} \widetilde{u}^{2}+2 \operatorname{sn}^{\prime}(p) u \widetilde{u}-u^{2}-\widetilde{u}^{2}+\operatorname{sn}^{2}(p)\right)$,

$\mathscr{H}=Q 4(u, \widetilde{u}, \widehat{u}, \widehat{\widetilde{u}} ; p, q) Q 4(u, \widetilde{u}, \widehat{u}, \widehat{\widetilde{u}} ; p,-q)=0$. 
We note that for the ABS equations, the case $K=0$ in Proposition 2.1 corresponds to H-type equations in the ABS list; for $\mathrm{H}$-type equations $P$ is a constant and for $\mathrm{H} 1$ even $P=0$; for A-type and Q-type equations, $Q$ and $P$ differ only in the parameter $q$.

\section{Bäcklund transformations}

In Section 1 by $\mathrm{H} 3(\delta)$ as an example we have illustrated its decomposition can be used to construct a BT. Motivated by the decomposition (2.5) of the ABS equations, we consider the following system

$$
\begin{aligned}
& h(u, \widetilde{u}, p)=U \widetilde{U}, \\
& h(u, \widehat{u}, q)=U \widehat{U},
\end{aligned}
$$

where to meet the consistency w.r.t. $u$ we request $U$ satisfies certain quadrilateral equation

$$
F(U, \widetilde{U}, \widehat{U}, \widehat{\widetilde{U}} ; p, q)=0
$$

which we call $U$-equation for convenience. In fact, on one hand, since $u$ must be well defined by (3.1), the two equations in (3.1) must be compatible (i.e. $\widehat{\widetilde{u}}=\widetilde{\widehat{u}}$ ), which leads to the $U$-equation. On the other hand, for arbitrary $U$ the function $h$ defined by (3.1) satisfies (2.4). In a more general case, considering the following pair

$$
h(u, \widetilde{u}, U, \widetilde{U}, p)=0, h(u, \widehat{u}, U, \widehat{U}, q)=0,
$$

if without knowing any information of $u$-equation (1.1), only by the compatibility $\widehat{\widetilde{u}}=\widetilde{\widehat{u}}$ we can eliminate $u$ from (3.3) to get $U$-equation (3.2), and vice versa, to get $u$-equation (1.1), we call (3.3) and the related $u$-equation (1.1) and $U$-equation (3.2) a consistent triplet generated by (3.3) (cf. [24]). Obviously, such a consistent triplet is nothing but a special case of BTs.

As for generating solutions, we note that for $\mathrm{H} 2$ and $\mathrm{H} 3, u$ solved from (3.1) with corresponding $h$ 's will provide a solution to these two equations, while for the rest equations in the ABS list there is uncertainty. For example, for Q1, we do not know whether $u$ solves $\mathrm{Q} 1$ or $\mathrm{Q} 1(u, \widetilde{u}, \widehat{u}, \widehat{\widetilde{u}} ; p,-q)=0$. In this section, instead of finding solutions, we are more interested in considering (3.1) as a BT to connect $u$-equation (2.4) and $U$-equation. In the following subsections Sec.3.1,3.2, we start from a generic affine-linear polynomial

$$
h(u, \widetilde{u}, p)=s_{0}(p)+s_{1}(p) u+s_{2}(p) \widetilde{u}+s_{3}(p) u \widetilde{u},
$$

where $s_{i}(p)$ are functions of $p$, and then examine all possibility that admits a consistent triplet in which both $u$-equation and $U$-equation are CAC. We note that $h=0$ with (3.4) is a discrete Riccati equation, and the special case $s_{1}=s_{2}$ with $s_{3}=0$ was already considered in [3].

\subsection{Consistent triplets}

When $h$ is defined as (3.4), for the relation of $h$ and possible forms of $u$-equation and $U$-equation, we have the following. 
Theorem 3.1. When $h(u, \widetilde{u}, p)$ in system (3.1) is defined by (3.4), then $U$-equation (3.2) is affinelinear if and only if either

$$
h(u, \widetilde{u}, p)=s_{0}(p)+s_{1}(p) u+s_{2}(p) \widetilde{u}
$$

or (after a constant shift $u \rightarrow u-c$ )

$$
h(u, \tilde{u}, p)=s_{0}(p)+s_{3}(p) u \widetilde{u} .
$$

Proof. When $h(u, \widetilde{u}, p)$ is defined by (3.4), we solve out from (3.1) that

$$
\widetilde{u}=\frac{U \widetilde{U}-s_{0}(p)-s_{1}(p) u}{s_{2}(p)+s_{3}(p) u}, \widehat{u}=\frac{U \widehat{U}-s_{0}(q)-s_{1}(q) u}{s_{2}(q)+s_{3}(q) u} .
$$

From the consistency $\widehat{\widetilde{u}}=\widetilde{\widetilde{u}}$, we have the resulting equation for $U: f(U, \widetilde{U}, \widehat{U}, \widehat{\widetilde{U}}, u)=0$, which should be independent of $u$. Therefore the coefficient of $u$ in $f$ should be zero, which leads to

$$
s_{2}(q) s_{3}(p)-s_{3}(q) s_{1}(p)=s_{2}(p) s_{3}(q)-s_{3}(p) s_{1}(q)=0 .
$$

When $s_{3}=0$, (3.4) turns to be (3.5). In this case, the system (3.1) is a BT for $u$-equation

$$
\frac{s_{0}(p)+s_{1}(p) u+s_{2}(p) \widetilde{u}}{s_{0}(q)+s_{1}(q) u+s_{2}(q) \widehat{u}}=\frac{s_{0}(q)+s_{1}(q) \widetilde{u}+s_{2}(q) \widehat{\widetilde{u}}}{s_{0}(p)+s_{1}(p) \widehat{u}+s_{2}(p) \widehat{\widetilde{u}}}
$$

and $U$-equation

$$
U\left(s_{1}(q) \widetilde{U}-s_{1}(p) \widehat{U}\right)+\widehat{\widetilde{U}}\left(s_{2}(q) \widehat{U}-s_{2}(p) \widetilde{U}\right)+s_{0}(q)\left(s_{1}(p)+s_{2}(p)\right)-s_{0}(p)\left(s_{1}(q)+s_{2}(q)\right)=0
$$

Here we note that equation (3.8), (3.9) and the BT (3.1) compose a consistent triplet (cf. [24]), i.e. viewing the BT (3.1) as a two-component system, then the compatibility of each component yields a lattice equation of the other component which is in the triplet.

When function $s_{3} \neq 0$, we have

$$
c=\frac{s_{2}(q)}{s_{3}(q)}=\frac{s_{1}(p)}{s_{3}(p)}=\frac{s_{1}(q)}{s_{3}(q)}=\frac{s_{2}(p)}{s_{3}(p)},
$$

where $c$ is a constant independent of $p$ and $q$. It then follows that $s_{2}=s_{1}=c s_{3}$. Thus (3.4) yields

$$
h(u, \widetilde{u}, p)=s_{0}(p)-c^{2} s_{3}(p)+s_{3}(p)(u+c)(\widetilde{u}+c),
$$

which then reduces to (3.6) by redefine $s_{0}(p) \rightarrow s_{0}(p)+c^{2} s_{3}(p)$ and $u \rightarrow u-c$. Consequently the $u$-equation reads

$$
\left(s_{0}(p)+s_{3}(p) u \widetilde{u}\right)\left(s_{0}(p)+s_{3}(p) \widehat{\widetilde{u}}\right)=\left(s_{0}(q)+s_{3}(q) u \widehat{u}\right)\left(s_{0}(q)+s_{3}(q) \widehat{\vec{u}}\right)
$$

and $U$-equation reads

$$
\begin{aligned}
& \left(s_{3}^{2}(p)-s_{3}^{2}(q)\right) U \widetilde{U} \widehat{U} \widehat{\widetilde{U}}+U\left(s_{0}(p) s_{3}^{2}(q) \widetilde{U}-s_{0}(q) s_{3}^{2}(p) \widehat{U}\right) \\
& +\widehat{\widetilde{U}}\left(s_{0}(p) s_{3}^{2}(q) \widehat{U}-s_{0}(q) s_{3}^{2}(p) \widetilde{U}\right)=s_{0}^{2}(p) s_{3}^{2}(q)-s_{0}^{2}(q) s_{3}^{2}(p) .
\end{aligned}
$$

Note that replacing $s_{0}$ with $-\frac{s_{0}}{s_{3}}$ and $s_{3}$ with $\frac{1}{s_{3}}$, equation (3.10) becomes (3.11). Eqs.(3.10), (3.11) and (3.1) compose a consistent triplet as well. 
Now we have obtained four quadrilateral equations, (3.8), (3.9), (3.10) and (3.11), all of which are derived as a compatibility of (3.1). Among them, equation (3.8) with $s_{0}=1, s_{1}(p)=p-$ $a, s_{2}(p)=p+a$ can be considered as the Nijhoff-Quispel-Capel (NQC) equation with $b=a$ (cf. [19] and eq.(9.49) in [10]).

\subsection{CAC property with $h$ given in (3.5) and (3.6)}

Although (3.8), (3.9), (3.10) and (3.11) are derived as a compatibility of (3.1), it is not true that they are CAC for arbitrary $s_{i}$. After a case-by-case investigation of the CAC property of the four equations, we reach a full list that includes all CAC equations when $h$ are given in (3.5) and (3.6), which is presented in the following theorem:

Theorem 3.2. For the system (3.1) where $h$ is affine-linear as given in (3.5) and (3.6), if it generates a consistent triplet and acts as a BT between quadrilateral equations which are CAC, the exhausted results are

Table 1. Consistent triplets.

\begin{tabular}{cccc}
\hline No. & $\mathrm{BT}(3.1)$ & $u$-equation & $U$-equation \\
\hline 1 & $\frac{1}{p}(u-\widetilde{u})=U \widetilde{U}$ & $\mathrm{Q} 1\left(0 ; p^{2}, q^{2}\right)$ & $1 \mathrm{pmKdV}$ \\
2 & $u+\widetilde{u}+p=U \widetilde{U}$ & $\mathrm{H} 2$ & $\mathrm{H} 1(2 p, 2 q)$ \\
3 & $\frac{1}{p}(u+\widetilde{u})-\delta p=U \widetilde{U}$ & $\mathrm{~A} 1\left(\delta ; p^{2}, q^{2}\right)$ & $\mathrm{H} 3(\delta ; 2 p, 2 q)$ \\
4 & $u \widetilde{u}+\delta p=U \widetilde{U}$ & $\mathrm{H} 3(\delta)$ & $\mathrm{H} 3(-\delta)$ \\
5 & $\frac{1}{p}(u \widetilde{u}-1)=U \widetilde{U}$ & $(\mathrm{~A} .11)$ & $\mathrm{H} 3(1)$ with $U \rightarrow U^{-1}$ \\
6 & $\frac{1}{\sqrt{1-p^{2}}}(1-p u \widetilde{u})=U \widetilde{U}$ & $\mathrm{~A} 2$ & $\mathrm{~A} 2\left(\sqrt{1-p^{2}}, \sqrt{1-q^{2}}\right)$ \\
\hline
\end{tabular}

Proof of the theorem is given in Appendix A.

Here we have two remarks. First, most of the BTs in Table 1 can be found from known literatures. For example, transformation 1 was already known in $[16,18,20]$ as a BT connecting the lattice Schwarzian KdV (Q1(0)) equation and lpmKdV equation, transformation 2 and 3 were given in [3], transformation 6 can be found from eq.(57) of [6] by first taking $(A, B, C)=(1,0,0)$, then $(0,0,1)$ and next eliminating $s, t$ from the obtained equations, transformation 4 can be found from eq.(58) of [6] by first taking $(A, B, C)=(0,1,0)$, then $(0,0,1)$ and next eliminating $s, t$ from the obtained equations. The second remark is although transformation 1 is a special case of 3 by taking $\delta=0$ and imposing a point transformation, we would like to keep it in the table because it does not only connect the lattice Schwarzian KdV equation and lpmKdV equation but also plays a practical role in deriving lattice equations in Cauchy matrix approach [17] as well as in generating rational solutions [24].

\subsection{Other cases: $Q 1(\delta), A 1(\delta)$ and $A 2$}

For equations $\mathrm{Q} 1(\delta), \mathrm{A} 1(\delta)$ and $\mathrm{A} 2$, their $h$ polynomials are not affine linear. We discuss them one by one. 
First, for Q1 $(\boldsymbol{\delta})$, the corresponding system (3.1) is

$$
(\widetilde{u}-u)^{2}-\delta^{2} p^{2}=p U \widetilde{U},(\widehat{u}-u)^{2}-\delta^{2} q^{2}=q U \widehat{U},
$$

which is quadratic w.r.t. $u$. We find if $u$ satisfies $\mathrm{Q} 1(\boldsymbol{\delta})$, then $U$ satisfies

$$
[p(U \widetilde{U}-\widehat{U} \widehat{\widetilde{U}})-q(U \widehat{U}-\widetilde{U} \widetilde{\widetilde{U}})]^{2}+4 p q(\widetilde{U}-\widehat{U})\left[\delta^{2}(p-q)(U-\widehat{\widetilde{U}})-U \widehat{\widetilde{U}}(\widetilde{U}-\widehat{U})\right]=0,
$$

and vice versa. The above equation can be transformed to $\mathrm{H} 3 *(\delta)$ equation

$$
(p-q)\left[p(U \widehat{U}-\widetilde{U} \widehat{\widetilde{U}})^{2}-q(U \widetilde{U}-\widehat{U} \widehat{\widetilde{U}})^{2}\right]+(U-\widehat{\widetilde{U}})(\widetilde{U}-\widehat{U})\left[(U-\widehat{\widetilde{U}})(\widetilde{U}-\widehat{U}) p q-4 \delta^{2}(p-q)\right]=0,
$$

by transformation $p \rightarrow 1 / p, q \rightarrow 1 / q$. Here we note that $\mathrm{H} 3 *(\delta)$ is one of integrable quad equations that are multi-quadratic counterparts of the ABS equations. These multi-quadratic equations are consistent in multi-dimensions as well and were systematically found in a beautiful work [6]. System (3.12) provides a BT between $\mathrm{Q} 1(\boldsymbol{\delta})$ and $\mathrm{H} 3 *(\boldsymbol{\delta})(3.13)$.

Similarly, for A1, we find

$$
(\widetilde{u}+u)^{2}-\delta^{2} p^{2}=p U \widetilde{U},(\widehat{u}+u)^{2}-\delta^{2} q^{2}=q U \widehat{U},
$$

provides a BT between $\mathrm{A} 1$ and $\mathrm{H} 3 *(\delta)(3.13)$.

For A2, in the system (3.1) there is

$$
h(u, \widetilde{u}, p)=\frac{(p u \widetilde{u}-1)(u \widetilde{u}-p)}{1-p^{2}} .
$$

It is hard to write out a $U$-equation in a neat form. However, by observing that $U$ is arbitrary in (3.1), we can replace $U$ with $U / f(u)$ where $f(u)$ is a suitable function of $u$ so that the deformed BT

$$
h(u, \widetilde{u}, p) f(u) f(\widetilde{u})=U \widetilde{U}, h(u, \widehat{u}, q) f(u) f(\widehat{u})=U \widehat{U}
$$

generates a $U$-equation with an explicit and neat form. Taking $f(u)=1 / u,(3.1)$ with (3.16) becomes

$$
\frac{(p u \widetilde{u}-1)(u \widetilde{u}-p)}{\left(1-p^{2}\right) u \widetilde{u}}=U \widetilde{U}, \frac{(q u \widehat{u}-1)(u \widehat{u}-q)}{\left(1-q^{2}\right) u \widehat{u}}=U \widetilde{U},
$$

which connects the solutions between $\mathrm{A} 2(u)$ and $\mathrm{A} 2 *(U)$ equation [6]

$$
\begin{aligned}
& (p-q)\left[p(U \widehat{U}-\widetilde{U} \widehat{\widetilde{U}})^{2}-q(U \widetilde{U}-\widehat{U} \widehat{\widetilde{U}})^{2}\right] \\
& \quad+(U-\widehat{\widetilde{U}})(\widetilde{U}-\widehat{U})[(U-\widehat{\widetilde{U}})(\widetilde{U}-\widehat{U})(p q-1)+2(p-q)(1+U \widetilde{U} \widehat{U} \widehat{\widetilde{U}})]=0
\end{aligned}
$$

with $p \rightarrow 2\left(p^{2}+1\right) /\left(1-p^{2}\right), q \rightarrow 2\left(q^{2}+1\right) /\left(1-q^{2}\right)$. One more example of utilizing $f(u)$ is $\mathrm{H} 3(\delta)$. For the transformation 4 in Table 1, taking $f(u)=u$ we have

$$
(u \widetilde{u}+\delta p) u \widetilde{u}=U \widetilde{U},(u \widehat{u}+\delta q) u \widehat{u}=U \widehat{U},
$$

which connects the solutions between $\mathrm{H} 3(\delta)(u)$ and $\mathrm{H} 3 *(\delta)(U)$ equation with parameters $p \rightarrow$ $4 / p^{2}, q \rightarrow 4 / q^{2}$. BTs (3.18) and (3.20) have been listed in [6]. Let us look at a third example. It can 
be verified that

$$
\frac{u \widetilde{u}-p}{p u \widetilde{u}-1}=U \widetilde{U}, \frac{u \widetilde{u}-q}{q u \widetilde{u}-1}=U \widehat{U}
$$

is an auto-BT of A2. Taking $f(u)=1 / u$ the new BT provides a transformation between $\mathrm{A} 2(u)$ and $\mathrm{A} 2 *(U)$ with parameters $p \rightarrow 2 p^{2}-1, q \rightarrow 2 q^{2}-1$.

We collect the BTs obtained in this subsection in Table 2.

Table 2. BT(3.1) related to $\mathrm{Q} 1(\delta), \mathrm{A} 1(\delta), \mathrm{H} 3(\delta)$ and $\mathrm{A} 2$.

\begin{tabular}{cccc}
\hline No. & $\mathrm{BT}(3.1)$ & $u$-equation & $U$-equation \\
\hline 1 & $\frac{1}{p}(\widetilde{u}-u)^{2}-\delta^{2} p=U \widetilde{U}$ & $\mathrm{Q} 1(\delta)$ & $\mathrm{H} 3 *\left(\delta ; \frac{1}{p}, \frac{1}{q}\right)$ \\
2 & $\frac{1}{p}(\widetilde{u}+u)^{2}-\delta^{2} p=U \widetilde{U}$ & $\mathrm{~A} 1(\delta)$ & $\mathrm{H} 3^{*}\left(\delta ; \frac{1}{p}, \frac{1}{q}\right)$ \\
3 & $\frac{(p u \widetilde{u}-1)(u \widetilde{u}-p)}{\left(1-p^{2}\right) u \widetilde{u}}=U \widetilde{U}$ & $\mathrm{~A} 2$ & $\mathrm{~A} 2 *\left(\frac{2\left(p^{2}+1\right)}{1-p^{2}}, \frac{2\left(q^{2}+1\right)}{1-q^{2}}\right)$ \\
4 & $(u \widetilde{u}+\delta p) u \tilde{u}=U \widetilde{U}$ & $\mathrm{H} 3(\delta)$ & $\mathrm{H} 3 *\left(\delta ; \frac{4}{p^{2}}, \frac{4}{q^{2}}\right)$ \\
5 & $\frac{u \widetilde{u}-p}{p u \widetilde{u}-1}=U \widetilde{U}$ & $\mathrm{~A} 2$ & $\mathrm{~A} 2$ \\
6 & $\frac{u \tilde{u}-p}{(p u \widetilde{u}-1) u \widetilde{u}}=U \widetilde{U}$ & $\mathrm{~A} 2$ & $\mathrm{~A} 2 *\left(2 p^{2}-1,2 q^{2}-1\right)$ \\
\hline
\end{tabular}

In this section we have given an exhausted examination for the case where $h$ is the affine-linear polynomial (3.4). For $\mathrm{Q} 1(\boldsymbol{\delta}), \mathrm{A} 1(\boldsymbol{\delta})$ and $\mathrm{A} 2$, their $h$ polynomials are not affine linear and their corresponding $U$-equations are usually multi-quadratic counterparts of the ABS equations, (see Table 2). For Q2, Q3 and Q4, their $h$ polynomials are so complicated that from system (3.1) we can not derive explicit $U$-equations.

We also note that there are many systematical works to consider constructions of BTs for quadrilateral equations $[3,6,13,14]$. In [6] many BTs are constructed by considering compatibility of (3.3) which are Riccati equations in terms of $U$ but allowing more freedom for $u$. Besides, [6] presents variety of BTs with free parameters $(A, B, C)$ that are derived by using Yang-Baxter maps. BTs in Table 2 are included in the results of [6].

\section{Applications}

BTs have been used as a main tool to find rational solutions for quadrilateral equations (see [24]). In this section we would like to introduce more applications, including a BT and weak Lax pair of $\mathrm{Q} 1(0)$, polynomial solutions of Q1 $(\boldsymbol{\delta})$ and rational solutions of $\mathrm{H}^{*}(\boldsymbol{\delta})$.

\subsection{BT and weak Lax pair of Q1(0)}

From the previous discussion, we know that Q1(0),

$$
p^{2}(u-\widehat{u})(\widetilde{u}-\widehat{\widetilde{u}})-q^{2}(u-\widetilde{u})(\widehat{u}-\widehat{\widetilde{u}})=0,
$$

has a BT

$$
\widetilde{u}-u=p U \widetilde{U}, \widehat{u}-u=q U \widehat{U}
$$


where $U$ satisfies the lpmKdV equation. If $U$ solves the lpmKdV equation, so does $1 / U$. Employing this symmetry we introduce

$$
\widetilde{\bar{u}}-\bar{u}=p U^{-1} \widetilde{U}^{-1}, \quad \widehat{\bar{u}}-\bar{u}=q U^{-1} \widehat{U}^{-1}
$$

as an adjoint system of (4.2), which is also a BT between Q1(0) and lpmKdV. Eliminating $U$ from (4.2) and (4.3) we reach

$$
(\widetilde{\bar{u}}-\bar{u})(\widetilde{u}-u)=p^{2}, \quad(\widehat{\bar{u}}-\bar{u})(\widehat{u}-u)=q^{2},
$$

which gives an auto-BT of Q1(0). Noticing the symmetry that $u$ and $1 / u$ can solve Q1(0) simultaneously, we replace $\bar{u}$ with $1 / \bar{u}$ and reach

$$
(\widetilde{\bar{u}}-\bar{u})(\widetilde{u}-u)+p^{2} \bar{u} \widetilde{\bar{u}}=0, \quad(\widehat{\bar{u}}-\bar{u})(\widehat{u}-u)+q^{2} \bar{u} \widehat{\bar{u}}=0,
$$

which gives another auto-BT of Q1(0). One can check that the following 6 equations

$$
\begin{aligned}
& p^{2}(u-\widehat{u})(\widetilde{u}-\widehat{\widetilde{u}})-q^{2}(u-\widetilde{u})(\widehat{u}-\widehat{\widetilde{u}})=0, \\
& (\widetilde{\bar{u}}-\bar{u})(\widetilde{u}-u)+p^{2} \bar{u} \widetilde{\bar{u}}=0, \\
& (\widehat{\bar{u}}-\bar{u})(\widehat{u}-u)+q^{2} \bar{u} \widehat{\bar{u}}=0, \\
& (\widehat{\bar{u}}-\widehat{\bar{u}})(\widehat{\widetilde{u}}-\widehat{u})+p^{2} \widehat{\bar{u}} \widehat{\bar{u}}=0, \\
& (\widehat{\widetilde{\bar{u}}}-\widetilde{\bar{u}})(\widehat{\widetilde{u}}-\widetilde{u})+q^{2} \widetilde{\bar{u}} \widehat{\bar{u}}=0, \\
& p^{2}(\bar{u}-\widehat{\bar{u}})(\widetilde{\bar{u}}-\widehat{\widetilde{\bar{u}}})-q^{2}(\bar{u}-\widetilde{\bar{u}})(\widehat{\bar{u}}-\widehat{\widetilde{\bar{u}}})=0
\end{aligned}
$$

can be consistently embedded on 6 faces of a cube.

The BT (4.3) yields a pair of linear problems (Lax pair):

$$
\widetilde{\Phi}=\left(\begin{array}{cc}
1 & 0 \\
\frac{p^{2}}{\tilde{u}-u} & 1
\end{array}\right) \Phi, \widehat{\Phi}=\left(\begin{array}{cc}
1 & 0 \\
\frac{q^{2}}{u}-u & 1
\end{array}\right) \Phi,
$$

where $\Phi=(g, f)^{T}$. The consistency of (4.6) leads to an equation

$$
(u-\widetilde{u}-\widehat{u}+\widehat{\widetilde{u}})\left[p^{2}(u-\widehat{u})(\widetilde{u}-\widehat{\widetilde{u}})-q^{2}(u-\widetilde{u})(\widehat{u}-\widehat{\widetilde{u}})\right]=0,
$$

which is Q1 $(0)$ multiplied by a factor $(u-\widetilde{u}-\widehat{u}+\widehat{\widetilde{u}})$. This means Q1(0) can not be fully determined by (4.6). Such an Lax pair is called a weak Lax pair and was first systematically studied in [11]. (4.6) is a new weak Lax pair of Q1(0). As a result, replacing (4.5a) and (4.5f) by

$$
u-\widetilde{u}-\widehat{u}+\widehat{\widetilde{u}}=0, \quad \bar{u} \widetilde{u} \widehat{\bar{u}} \widehat{\bar{u}}(1 / \bar{u}-1 / \widetilde{\bar{u}}-1 / \widehat{\bar{u}}+1 / \widehat{\widetilde{u}})=0,
$$

respectively, (4.5) is also consistent around the cube as a system.

In addition to the weak Lax pair of Q1(0), we have shown an approach to construct auto-BT for $u$-equation from (3.1) if $U$-equation admits a symmetry $U \rightarrow 1 / U$. For A2 and related BT (3.21), 
employing the same technique, we have relations

$$
(u \widetilde{u}-p)(\bar{u} \widetilde{\bar{u}}-p)=(p u \widetilde{u}-1)(p \bar{u} \widetilde{\bar{u}}-1), \quad(u \widehat{u}-q)(\bar{u} \widehat{\bar{u}}-q)=(q u \widehat{u}-1)(q \bar{u} \widehat{\bar{u}}-1),
$$

and

$$
(u \widetilde{u}-p)(1-p \bar{u} \widetilde{\bar{u}})=(p u \widetilde{u}-1)(p-\bar{u} \widetilde{\bar{u}}), \quad(u \widehat{u}-q)(1-q \widehat{u} \widehat{\bar{u}})=(q u \widehat{u}-1)(q-\bar{u} \widehat{\bar{u}}) .
$$

Both of them are auto BTs of A2.

\subsection{Polynomial solutions of $Q 1(\delta)$}

Consider (3.12), i.e.

$$
(\widetilde{u}-u)^{2}-\delta^{2} p^{2}=p U \widetilde{U},(\widehat{u}-u)^{2}-\delta^{2} q^{2}=q U \widehat{U},
$$

which is a BT between $\mathrm{Q} 1(\delta)$ and $\mathrm{H} 3 *(\delta)$. However, if we do not care about what $U$-equation is, then from decomposition (2.5f) any $u$ defined by (4.8) will be a solution of

$$
\mathscr{H}=Q 1(u, \widetilde{u}, \widehat{u}, \widehat{\widetilde{u}} ; p, q) Q 1(u, \widetilde{u}, \widehat{u}, \widehat{\widetilde{u}} ; p,-q)=0 .
$$

In other words, (4.8) may also be a BT between $Q 1(u, \widetilde{u}, \widehat{u}, \widehat{\widetilde{u}} ; p,-q)=0$ and some $U$-equation other than $\mathrm{H} 3 *(\delta)$. This means, if we just solve (4.8) and obtain $u$, we should verify whether $u$ satisfies $\mathrm{Q} 1(\delta)(1.3 \mathrm{f})$ or $Q 1(u, \widetilde{u}, \widehat{u}, \widehat{\widetilde{u}} ; p,-q)=0$.

To solve (4.8) which is a quadratic system, we suppose that $U$ is a polynomial of

$$
x=a n+b m+\gamma,
$$

say,

$$
U=\sum_{i=0}^{N} c_{N-i} x^{i}
$$

with constants $a, b, \gamma, c_{i}$ and $c_{0} \neq 0, N \geq 0$. Introduce

$$
v_{1}=\widetilde{u}-u, \quad v_{2}=\widehat{u}-u,
$$

where $v_{1}$ and $v_{2}$ should satisfy

$$
\widehat{v}_{1}-v_{1}=\widetilde{v}_{2}-v_{2}
$$

due to consistency of (4.12). For the case that both $v_{1}$ and $v_{2}$ are also polynomials of $x$, we have the following result:

Theorem 4.1. When $U$ is given in (4.11), we can convert the system (4.8) to

$$
v_{1}^{2}-\delta^{2} p^{2}=p U \widetilde{U}, v_{2}^{2}-\delta^{2} q^{2}=q U \widehat{U} .
$$

When $N \geq 1$ and we require that $v_{i}$ have the following form,

$$
v_{1}=\sum_{i=0}^{N} f_{N-i} x^{i}, v_{2}=\sum_{i=0}^{N} g_{N-i} x^{i}
$$

with constants $f_{i}, g_{i}$ to be determined, the only allowed values for $N$ are 1 and $2 . u$ is recovered through (4.12). 
The proof for this theorem is given in Appendix B.

Let us turn to find polynimial solutions. When $N=0$, we have $U=c_{0}$ and

$$
(\widetilde{u}-u)^{2}=p\left(\delta^{2} p+c_{0}^{2}\right),(\widehat{u}-u)^{2}=q\left(\delta^{2} q+c_{0}^{2}\right) .
$$

Suppose

$$
p=\frac{c_{0}^{2}}{a^{2}-\delta^{2}}, \quad q=\frac{c_{0}^{2}}{b^{2}-\delta^{2}}, \quad \alpha=p a, \beta=q b .
$$

It turns out that four possibilities for $u$ are

$$
\begin{aligned}
& \alpha n+\beta m+\gamma, \quad \frac{(-1)^{n+1}}{2} \alpha+\beta m+\gamma, \\
& \alpha n+\frac{(-1)^{m+1}}{2} \beta+\gamma, \frac{(-1)^{n+1}}{2} \alpha+\frac{(-1)^{m+1}}{2} \beta+\gamma,
\end{aligned}
$$

which coincide with the result in [12].

When $N=1,2$, with $p, q$ parameterized as

$$
p=a^{2}, \quad q=b^{2},
$$

following Theorem 4.1, after some calculation and scaling, we find solutions to (4.8):

$$
\begin{aligned}
& u= \pm \delta x^{2}+\gamma_{0}, \quad U= \pm 2 \delta x, \\
& u=\frac{c_{0}}{3} x^{3}-\frac{\delta^{2}}{c_{0}} x-\frac{c_{0}}{3}\left(a^{3} n+b^{3} m\right)+\gamma_{0}, \quad U=c_{0} x^{2}-\frac{\delta^{2}}{c_{0}} .
\end{aligned}
$$

We can check that $u$ and $U$ respectively satisfy $\mathrm{Q} 1(\delta)$ and $\mathrm{H} 3 *(\delta)$ equation (3.13). These are polynomial solutions.

\subsection{Rational solutions of $\mathrm{H} 3 *(\delta)$}

One can derive rational solutions for $\mathrm{H}^{*}(\boldsymbol{\delta})$ from those of $\mathrm{Q} 1(\boldsymbol{\delta})$ and $\mathrm{BT}(3.12)$.

It has been proved that $\mathrm{Q} 1(\delta)$ with $p, q$ parameterized as in (4.17) has the following rational solutions [24]:

$$
u_{N+2}=\frac{\overline{\bar{f}}+\delta^{2} \underline{\underline{f}}}{f},
$$

where $N$-th order Casoratian $f$ is given by

$$
f \doteq f_{N}=|\widehat{N-1}|=|\alpha(n, m, 0), \alpha(n, m, 1), \cdots, \alpha(n, m, N-1)|,
$$

for $N \geq 1$ and extended to negative direction by

$$
f_{-N}=(-1)^{\left[\frac{N}{2}\right]} f_{N-1}, \quad f_{0}=1,
$$

$[\cdot]$ denotes the greatest integer function, $\underline{f}=f_{N-1}=|\widehat{N-2}|, \bar{f}=f_{N+1}=|\widehat{N}|$, etc; the Casoratian vector $\alpha$ is

$$
\alpha(n, m, l)=\left(\alpha_{0}, \alpha_{1}, \cdots, \alpha_{M-1}\right)^{T}, \quad \alpha_{j}=\left.\frac{1}{(2 j+1) !} \partial_{s_{i}}^{2 j+1} \psi_{i}\right|_{s_{i}=0}, \quad M=1,2, \cdots
$$

with function $\psi_{i}$ 


$$
\psi_{i}(n, m, l)=\psi_{i}^{+}(n, m, l)+\psi_{i}^{-}(n, m, l), \quad \psi_{i}^{ \pm}(n, m, l)=\rho_{i}^{ \pm}\left(1 \pm s_{i}\right)^{l}\left(1 \pm a s_{i}\right)^{n}\left(1 \pm b s_{i}\right)^{m},
$$

where

$$
\rho_{i}^{ \pm}= \pm \frac{1}{2} \exp \left[-\sum_{j=1}^{\infty} \frac{\left(\mp s_{i}\right)^{j}}{j} \gamma_{j}\right], \gamma_{j} \in \mathbb{C}
$$

The Casoratian $f$ defined above satisfies a superposition relation [24]

$$
\begin{aligned}
& \widetilde{\overline{\bar{f}}} f-\overline{\bar{f}} \tilde{f}=a \widetilde{\bar{f}} \bar{f}, \\
& \widehat{\overline{\bar{f}}} f-\overline{\bar{f}} \widehat{f}=b \overline{\bar{f}} \bar{f} .
\end{aligned}
$$

Making use of (3.12), (4.19), (4.20) and (4.21), by a direct calculation we find rational solutions of $\mathrm{H}^{*}(\delta)(3.13)$ can be written as

$$
U_{N+2}=\frac{\bar{f}^{2}-\delta^{2} \underline{f}^{2}}{f^{2}}, N \in \mathbb{Z} .
$$

The first three solutions are

$$
\begin{aligned}
& U_{1}=1-x_{1}^{2} \delta^{2}, \\
& U_{2}=x_{1}^{2}-\delta^{2}, \\
& U_{3}=\frac{\left(x_{1}^{3}-x_{3}\right)^{2}-9 \delta^{2}}{9 x_{1}^{2}},
\end{aligned}
$$

where

$$
x_{i}=a^{i} n+b^{i} m+\gamma_{i}
$$

Here $U_{2}$ is $(4.18 \mathrm{~b})$ with $c_{0}=1$.

Finally, we note that, compared with the solution of $\mathrm{H} 3(\delta)$ given by [24], which is

$$
u_{N+2}=(-1)^{\frac{n+m}{2}+\frac{1}{4}} \frac{\bar{f}+(-1)^{n+m} \boldsymbol{\delta} \underline{f}}{f},
$$

when $\delta=i=\sqrt{-1}$ it is interesting to find the relation $U_{N}=\left|u_{N}\right|^{2}$.

\section{Conclusions}

BTs contain compatibility and are closely related to integrability of the equations that they connect. In this paper we have investigated system (3.1) as a BT. When $h$ is affine linear with a generic form (3.4), we made a complete examination and all consistent triplets are listed in Table 1. As applications, apart from constructing solutions (cf. [24]), these BTs in the triplets can be viewed as Lax pairs of $u$-equations, where wave function $\Phi=(g, f)^{T}$ can be introduced by taking $U=g / f$ but usually it is hard to introduce an significant spectral parameter. When $h$ is beyond affine linear, system (3.1) as a BT and the connecting quadrilateral equations (including multi-quadratic ones) are listed in Table 2. Further applications of the obtained BTs, such as constructing weak Lax pair and rational solutions for multi-quadratic lattice equations, were also shown in the paper. 


\section{Acknowledgments}

We are grateful to the referees and editor for their invaluable comments. This project is supported by the NSF of China (Nos.11371241, 11631007 and 11601312).

\section{Appendix A. Proof of Theorem 3.2}

\section{A.1. Multidimensional consistency: $h$ given in (3.5)}

The following discussion is on the basis of the CAC condition $\overline{\widetilde{\widetilde{u}}}=\widehat{\widetilde{\widetilde{u}}}=\widetilde{\widehat{\vec{u}}}$ for system (1.2). First we investigate the case of (3.8) and (3.9) with $h(u, \widetilde{u}, p)$ given in (3.5) where we assume $s_{1}(p) s_{2}(p) \neq 0$, otherwise (3.8) is not a quadrilateral equation.

\section{A.1.1. $s_{0}=0$}

In this case, it can be verified that (3.8) always satisfies the CAC condition $\overline{\widetilde{u}}=\widehat{\widetilde{u}}=\widetilde{\widehat{u}}$. Canonically, we make a transformation $u \rightarrow\left(-\frac{s_{1}(p)}{s_{2}(p)}\right)^{n}\left(-\frac{s_{1}(q)}{s_{2}(q)}\right)^{m} u$ so that equation (3.8) is in a neat form

$$
s_{1}(p) s_{2}(p)(u-\widetilde{u})(\widehat{u}-\widehat{\widetilde{u}})=s_{1}(q) s_{2}(q)(u-\widehat{u})(\widetilde{u}-\widehat{\widetilde{u}}) .
$$

Without any loss of generality, by assumption of $s_{1}(p)=-s_{2}(p)=\frac{1}{p}$, equation (3.8) turns out to be the equation $\mathrm{Q} 1\left(0 ; p^{2}, q^{2}\right)^{\mathrm{a}}$, while the corresponding equation (3.9) becomes the lpmKdV equation,

$$
p(U \widetilde{U}-\widehat{U} \widehat{\widetilde{U}})-q(U \widehat{U}-\widetilde{U} \widehat{\widetilde{U}})=0 .
$$

\section{A.1.2. $s_{0} \neq 0$}

A. $s_{1}(p)+s_{2}(p)=k s_{0}(p)$ with constant $k$

This goes to the case of $s_{0}=0$ by taking $u \rightarrow u-k^{-1}$ when $k \neq 0$ and $u \rightarrow u-s_{0}(p) n-s_{0}(q) m$ when $k=0$.

B. $s_{1}(p)+s_{2}(p)=k s_{0}(p)$ with nonconstant $k$

Check all terms in $\overline{\widehat{u}}=\widetilde{\widehat{u}}$, where the coefficient of $u \bar{u}^{3}$ reads

$$
s_{1}(p) s_{2}^{4}(r) A(B+C)
$$

with

$$
\begin{aligned}
A= & s_{2}(p) s_{1}(p)-s_{2}(q) s_{1}(q), \quad B=\left(-s_{0}(p) s_{1}(p)+s_{0}(q) s_{1}(q)\right)\left(s_{2}(r)+s_{1}(r)\right), \\
C= & \left(s_{1}^{2}(p)+s_{2}(p) s_{1}(p)-s_{1}^{2}(q)-s_{2}(q) s_{1}(q)\right) s_{0}(r) \\
& +\left[s_{0}(p) s_{2}(q)+s_{1}(q) s_{0}(p)-s_{1}(p) s_{0}(q)-s_{0}(q) s_{2}(p)\right] s_{1}(p) s_{1}(q) s_{2}^{-1}(r) .
\end{aligned}
$$

Letting (A.2) vanish leads to only three subcases.

Case B.1. $A=0$

$\overline{{ }^{\mathrm{a}} \mathrm{By} \text { this we denote } \mathrm{Q} 1(0)}$ in which replacing $p$ and $q$ by $p^{2}$ and $q^{2}$. 
It directly results in

$$
s_{2}(p) s_{1}(p)=c_{0}, \text { with constant } c_{0} .
$$

Then from the coefficient of $\bar{u}^{3}$ we have

$$
s_{1}^{4}(p) s_{1}^{2}(q) E F=0
$$

where

$$
\begin{aligned}
E= & s_{0}(p) s_{1}(p)\left(s_{1}^{2}(q)+c_{0}\right)-s_{0}(q) s_{1}(q)\left(s_{1}^{2}(p)+c_{0}\right), \\
F= & c_{0}^{2} s_{1}(p) s_{0}(p)-c_{0}^{2} s_{0}(q) s_{1}(q)-c_{0}\left(s_{1}^{2}(p)-s_{1}^{2}(q)\right) s_{0}(r) s_{1}(r) \\
& +\left(s_{0}(q) s_{1}(p)-s_{0}(p) s_{1}(q)\right) s_{1}(p) s_{1}(q) s_{1}^{2}(r) .
\end{aligned}
$$

If $E=0$, it returns to the Case A. In fact, when $E=0$, under (A.3) we have

$$
\frac{s_{1}^{2}(p)+c_{0}}{s_{1}(p) s_{0}(p)}=\frac{s_{1}^{2}(q)+c_{0}}{s_{1}(q) s_{0}(q)}=c_{1}=\frac{s_{1}(p)+s_{2}(p)}{s_{0}(p)},
$$

with constant $c_{1}$.

In the case that $F=0$ and $s_{1}(p)$ is not a constant, it again returns to Case A. In fact, in this case from $F=0$ we can take $s_{0}$ to be the form

$$
s_{0}(t)=c_{1} s_{1}(t)+c_{2} s_{1}^{-1}(t),
$$

where $c_{1}$ and $c_{2}$ are constants. Substituting the above with $t=p, q, r$ into $F=0$ it turns out that $c_{2}=c_{1} c_{0}$, from which and (A.3) we find $s_{1}(p)+s_{2}(p)=s_{0}(p) / c_{1}$, which brings the case to Case A. Thus the only choice is $s_{1}(p)$ being a constant. Without loss of generality we suppose $s_{1}(p)=1$ and as a consequence of (A.3) we also have $s_{2}(p)=c_{0}$. Then, after checking the remaining terms in $\overline{\widehat{u}}=\widehat{\bar{u}}=\widetilde{\widehat{u}}$ we find $c_{0}=1$. Therefore in this case we have $h(u, \widetilde{u}, p)=u+\widetilde{u}+p$, and (3.8) and (3.9) are nothing but $\mathrm{H} 2$ and $\mathrm{H} 1(2 p, 2 q)$.

Case B.2. $A \neq 0, B=C=0$

$B=0$ yields either $s_{2}(r)+s_{1}(r)=0$ or $s_{0}(p) s_{1}(p)=c_{0}$ with constant $c_{0}$. The former belongs to Case $\mathrm{A}$ and then we consider the later, i.e.

$$
s_{0}(p) s_{1}(p)=c_{0} .
$$

Note that if the term $s_{1}^{2}(p)+s_{2}(p) s_{1}(p)-s_{1}^{2}(q)-s_{2}(q) s_{1}(q)$ in $C$ vanishes we will find $\left(s_{1}(p)+\right.$ $\left.s_{2}(p)\right) s_{1}(p)$ to be a constant, which, together with (A.5), again leads to Case A. If the term does not vanish, from $C=0$ we can assume there are constants $c_{2}$ and $c_{3}$ such that $c_{2} s_{0}(r)+c_{3} s_{2}^{-1}(r)=0$, i.e.

$$
s_{0}(r) s_{2}(r)=c_{1}=-c_{3} / c_{2} .
$$

Making use of (A.5) and (A.6) we reach

$$
\frac{c_{0}\left(c_{1}^{2}-c_{0}^{2}\right)}{c_{1}}\left(\frac{1}{s_{0}^{2}(p)}-\frac{1}{s_{0}^{2}(q)}\right)=0 .
$$

We ignore solution $s_{0}=c$ because this leads to $s_{1}$ and $s_{2}$ to be constants and then brings the case to Case A. Therefore we have $c_{1}= \pm c_{0}$. Since $c_{1}=-c_{0}$ results in $k=0$ which is Case A, the only 
choice is $c_{1}=c_{0}$ and in this case the canonical form for $h$ can be

$$
h(u, \widetilde{u}, p)=\frac{1}{p}(\widetilde{u}+u)-\delta p .
$$

Then it follows that (3.8) is $\mathrm{A} 1\left(\delta ; p^{2}, q^{2}\right)$, and the corresponding (3.9) is $\mathrm{H} 3(\delta ; 2 p, 2 q)$.

Case B.3. $A B \neq 0, B+C=0$

Since $B \neq 0$, from $B+C=0$ we can assume

$$
s_{1}(r)+s_{2}(r)=c_{1} s_{0}(r)+c_{2} s_{2}^{-1}(r),
$$

with constant $c_{1}$ and nonzero constant $c_{2}$ (if $c_{2}=0$ we back to Case $\mathrm{A}$ ), in which

$$
\begin{aligned}
& c_{1}=\frac{s_{1}(p)\left(s_{2}(p)+s_{1}(p)\right)-s_{1}(q)\left(s_{2}(q)+s_{1}(q)\right)}{s_{0}(p) s_{1}(p)-s_{0}(q) s_{1}(q)}, \\
& c_{2}=\frac{\left[s_{0}(p)\left(s_{2}(q)+s_{1}(q)\right)-s_{0}(q)\left(s_{1}(p)+s_{2}(p)\right)\right] s_{1}(p) s_{1}(q)}{s_{0}(p) s_{1}(p)-s_{0}(q) s_{1}(q)} .
\end{aligned}
$$

Note that in Case B $s_{0}(r)$ and $s_{2}^{-1}(r)$ must be linearly independent. Separate $p$ and $q$ in (A.8a) we find $s_{1}(p)\left(s_{2}(p)+s_{1}(p)\right)-c_{1} s_{0}(p) s_{1}(p)=c_{0}$ with nonzero constant $c_{0}$, which, together with relation (A.7), yields $s_{2}(p)=c_{2} c_{0}^{-1} s_{1}(p)$. Now, substituting this relation and (A.7) into (A.8b) we find $c_{2}=c_{0}$, and consequently, $s_{1}(p)=s_{2}(p)$. Thus, (3.5) of this case reads

$$
h(u, \widetilde{u}, p)=s_{0}(p)+s_{1}(p) u+s_{1}(p) \widetilde{u},
$$

and (A.7) reads

$$
2 s_{1}(r)=c_{1} s_{0}(r)+c_{0} s_{1}^{-1}(r) .
$$

We note that (A.9) is already discussed in [3]. After making $u \rightarrow u-1 / c_{1}$ in (A.9) we can consider

$$
h(u, \widetilde{u}, p)=-\frac{c_{0}}{c_{1} s_{1}(p)}+s_{1}(p) u+s_{1}(p) \widetilde{u},
$$

which, however, leads to Case B.2. So, nothing new is obtained in this case.

\section{A.2. CAC property: $h$ given in (3.6)}

For $h(u, \widetilde{u}, p)$ given in (3.6), we suppose $s_{0}(p), s_{3}(p) \neq 0$ and they are not constants simultaneously, so that we can keep the freedom of $p, q$. By the same manner, from the coefficient of $\widetilde{u} \widehat{u}^{3}$ in $\overline{\widehat{u}}=\widetilde{\widehat{u}}$, we find

$$
s_{3}^{2}(p) s_{0}^{2}(r)-s_{3}^{2}(r) s_{0}^{2}(p)+s_{3}^{2}(r) s_{0}^{2}(q)-s_{3}^{2}(q) s_{0}^{2}(r)+s_{3}^{2}(q) s_{0}^{2}(p)-s_{3}^{2}(p) s_{0}^{2}(q)=0 .
$$

If $s_{3}(p)$ is a constant, setting $s_{3}(p)=1, s_{0}(p)=p \delta$, we have $h(u, \widetilde{u}, p)=u \widetilde{u}+p \delta$, (3.10) is $\mathrm{H} 3(\boldsymbol{\delta})$ and $(3.11)$ is $\mathrm{H} 3(-\boldsymbol{\delta})$.

If $s_{0}(p)$ is a constant, by setting $s_{0}(p)=1, s_{3}(p)=p \delta$, it comes out that $h(u, \widetilde{u}, p)=\delta p u \widetilde{u}+1$. By transformation $u \rightarrow u^{-1},(3.10)$ also reaches $\mathrm{H} 3(\delta)$. 
If neither $s_{0}(p)$ or $s_{3}(p)$ is a constant, $\partial_{p} \partial_{q}(\mathrm{~A} .10)$ yields

$$
\frac{\left(s_{0}^{2}(p)\right)^{\prime}}{\left(s_{3}^{2}(p)\right)^{\prime}}=\frac{\left(s_{0}^{2}(q)\right)^{\prime}}{\left(s_{3}^{2}(q)\right)^{\prime}}=c_{1},
$$

which leads to $s_{0}^{2}(p)=c_{1} s_{3}^{2}(p)+c_{2}$ with nonzero constant $c_{1}$. When $c_{2}=0$, it yields $s_{0}(p)=\delta s_{3}(p)$. Replacing $u \rightarrow(-\delta)^{1 / 2} u$ and taking $s_{3}(p)=1 / p$, we can write equation (3.10) as

$$
q^{2}(u \widetilde{u}-1)(\widehat{u} \widehat{\bar{u}}-1)=p^{2}(u \widehat{u}-1)(\widehat{\widetilde{u}}-1),
$$

and the corresponding (3.11) is H3(1) after transformation $U \rightarrow U^{-1}$. When $c_{2} \neq 0$, we can scale $c_{1}$ to be 1 by replacing $u \rightarrow\left(c_{1}\right)^{1 / 4} u$. Then, setting $c_{2}=1, s_{3}(p)=\frac{-p}{\sqrt{1-p^{2}}}, s_{0}(p)=\frac{1}{\sqrt{1-p^{2}}}$, equation (3.10) reduces to $\mathrm{A} 2$ and (3.11) reduces to $\mathrm{A} 2\left(\sqrt{1-p^{2}}, \sqrt{1-q^{2}}\right)$.

We note that (A.11) is not a new equation. It is related to Q1 $\left(0 ; p^{2}, q^{2}\right)$ by $u \rightarrow u^{(-1)^{n+m}}$.

As a conclusion we have proved Theorem 3.2.

\section{Appendix B. Proof of Theorem 4.1}

According to the BT (4.8) and assumption (4.11) and (4.12), we can assume that $v_{i}$ have the following special form

$$
v_{1}=(-1)^{\theta_{1}} \sum_{i=0}^{N} f_{N-i} x^{i}, v_{2}=(-1)^{\theta_{2}} \sum_{i=0}^{N} g_{N-i} x^{i}
$$

with constants $f_{i}, g_{i}$ to be determined, where $\theta_{i}$ can be arbitrary functions of $n, m$. First we have the following.

Lemma B.1. With $U$ defined in (4.11) and $v_{1}, v_{2}$ defined above, when $N \geq 1$, we have

$$
v_{1}=\sum_{i=0}^{N} f_{N-i} x^{i}, \quad v_{2}=\sum_{i=0}^{N} g_{N-i} x^{i} .
$$

Proof. Substituting (4.11) and (B.1) into system (4.8) and (4.13), from the coefficient of the leading term $x^{2 N}$ (if $N \geq 1$ ) in (4.8) we find

$$
f_{0}^{2}=c_{0}^{2} p, g_{0}^{2}=c_{0}^{2} q,
$$

which means $\frac{f_{0}^{2}}{g_{0}^{2}}=\frac{p}{q}$, and from the coefficient of $x^{N}$ in (4.13) we find

$$
f_{0}\left((-1)^{\widehat{\theta}_{1}}-(-1)^{\theta_{1}}\right)=g_{0}\left((-1)^{\widetilde{\theta}_{2}}-(-1)^{\theta_{2}}\right) .
$$

Consequently we have

$$
\left((-1)^{\widehat{\theta}_{1}}-(-1)^{\theta_{1}}\right)^{2} p=\left((-1)^{\widetilde{\theta}_{2}}-(-1)^{\theta_{2}}\right)^{2} q .
$$

Since $p, q$ are independent constants, it follows that $\theta_{1}=\theta_{1}(n), \theta_{2}=\theta_{2}(m)$. Consequently, from the coefficient of $x^{N-1}(N \geq 1)$ in (4.13) we have $(-1)^{\theta_{1}} f_{0} b=(-1)^{\theta_{2}} g_{0} a$, which leads to the fact that $\theta_{1}$ and $\theta_{2}$ are constants. Noticing that $\theta_{1}$ and $\theta_{2}$ can be absorbed into $f_{i}, g_{i}$, we can assume $\theta_{1}=\theta_{2}=0$ without loss of generality. Thus (B.1) becomes (B.2). 
Lemma B.2. With $U$ defined in (4.11) and $v_{1}, v_{2}$ defined in (B.2), when $N \geq 1$, the allowed values of $N$ are only 1,2 .

Proof. Analyzing the coefficient of $x^{N-1}(N \geq 1)$ in (4.13), we obtain $f_{0} b=g_{0} a$, which leads to $\frac{a^{2}}{b^{2}}=\frac{p}{q}$ in light of (B.3). So we have $p=\theta a^{2}, q=\theta b^{2}$ with constant $\theta$. $\theta$ can be scaled to be 1 using $\delta$, therefore we set $p=a^{2}, q=b^{2}$ (i.e. (4.17)) and $f_{0}=t c_{0} a, g_{0}=t c_{0} b, t^{2}=1$ from (B.3). One can always take $t$ to be 1 as system (4.8) remains invariant under $U \rightarrow-U$. Consequently we have

$$
f_{0}=c_{0} a, g_{0}=c_{0} b .
$$

Substituting (B.2), (4.17) and (B.4) into the coefficient of $x^{2 N-1}(N \geq 1)$ in (4.8), we can work out

$$
f_{1}=\frac{2 c_{1}+c_{0} a N}{2} a, g_{1}=\frac{2 c_{1}+c_{0} b N}{2} b .
$$

Then substituting (4.17)-(B.5) into the coefficient of $x^{N-2}(N \geq 2)$ in (4.13), we find it varnishes. Next, analyzing the coefficient of $x^{2 N-2}(N \geq 2)$ in (4.8), we have

$$
\begin{aligned}
& f_{2}=\frac{a}{8}\left(8 c_{2}+4 a c_{1}(N-1)+a^{2} c_{0}\left(N^{2}-2 N\right)\right), \\
& g_{2}=\frac{b}{8}\left(8 c_{2}+4 b c_{1}(N-1)+b^{2} c_{0}\left(N^{2}-2 N\right)\right) .
\end{aligned}
$$

Substituting (4.17)-(B.6) into the coefficient of $x^{N-3}(N \geq 3)$ in (4.13), we obtain

$$
a b c_{0}\left(a^{2}-b^{2}\right)\left(N^{2}+2 N\right)=0,
$$

which admits zero option if $N \geq 3$. Therefore all possible choices of $N$ can only be 1,2 .

\section{References}

[1] V.E. Adler, A.I. Bobenko and Yu.B. Suris, Classification of integrable equations on quad-graphs. The consistency approach, Comm. Math. Phys. 233 (2003) 513-543.

[2] V.E. Adler, A.I. Bobenko and Yu.B. Suris, Discrete nonlinear hyperbolic equations. Classification of integrable cases, Funct. Anal. Appl. 43 (2009) 3-17.

[3] J. Atkinson, Bäcklund transformations for integrable lattice equations, J. Phys. A: Math. Theor 41 (2008) No.135202 (8 pp).

[4] J. Atkinson, Singularities of type-Q ABS equations, SIGMA 7 (2011) No.073 (14 pp).

[5] J. Atkinson and N. Joshi, Singular-boundary reductions of type-Q ABS equations, Int. Math. Res. Not. 2013 (2013) 1451-1481.

[6] J. Atkinson and M. Nieszporski, Multi-quadratic quad equations: Integrable cases from a factorizeddiscriminant hypothesis. Int. Math. Res. Not. 2014 (2013) 4215-4240.

[7] R. Boll, Classification of 3D consistent quad-equations, J. Nonl. Math. Phys. 18 (2011) 337-365.

[8] R. Boll, Classification and Lagrangian structure of $3 D$ consistent quad-equations, (Doctoral Thesis, Technischen Universität Berlin at Berlin, 2012), http://dx.doi.org/10.14279/depositonce-3302.

[9] J. Hietarinta, Searching for CAC-maps, J. Nonl. Math. Phys. 12 (2005) 207-214.

[10] J. Hietarinta, N. Joshi and F.W. Nijhoff, Discrete Systems and Integrablity, (Camb. Univ. Press, Cambridge, 2016).

[11] J. Hietarinta and C. Viallet, Weak Lax pairs for lattice equations, Nonlinearity 25 (2011) 1955-1966.

[12] J. Hietarinta and D.J. Zhang, Soliton solutions for ABS lattice equations. II. Casoratians and bilinearization, J. Phys. A: Math. Theor. 42 (2009) No.404006 (30 pp).

[13] P. Kassotakis and M. Nieszporski, Families of integrable equations, SIGMA 7 (2011) No.100 (14 pp). 
[14] P. Kassotakis and M. Nieszporski, On non-multiaffine consistent around the cube lattice equations, Phys. Lett. A 376 (2012) 3135-3140.

[15] R.M. Miura, Bäcklund Transformations, the Inverse Scattering Method, Solitons, and Their Applications: NSF Research Workshop on Contact Transformations, (Springer-Verlag, Berlin, Heidelberg, 1976).

[16] F.W. Nijhoff, On some "Schwarzian" equations and their discrete analogues, in Algebraic Aspects of Integrable Systems: In Memory of Irene Dorfman, Eds. A.S. Fokas, I. M. Gelfand, (Birkhliuser, Boston, 1997) 237-260.

[17] F.W. Nijhoff, J. Atkinson and J. Hietarinta, Soliton solutions for ABS lattice equations. I. Cauchy matrix approach, J. Phys. A: Math. Theor. 42 (2009) No.404005 (34pp).

[18] F.W. Nijhoff and H.W. Capel, The discrete Korteweg-de Vries equation, Acta Appl. Math. 39 (1995) 133-158.

[19] F.W. Nijhoff, G.R.W. Quispel and H.W. Capel, Direct linearization of nonlinear difference-difference equations. Phys. Lett. A 97 (1983) 125-128.

[20] F.W. Nijhoff, A. Ramani, B. Grammaticos and Y. Ohta, On discrete Painlevé equations associated with the lattice KdV systems and the Painlevé VI equation, Stud. Appl. Math. 106 (2001) 261-314.

[21] F.W. Nijhoff and A.J. Walker, The discrete and continuous Painlevé VI hierarchy and the Garnier systems, Glasg. Math. J. 43A (2001) 109-123.

[22] R. Prus and A. Sym, Rectilinear congruences and Bäcklund transformations: roots of the soliton theory, in Nonlinearity \& Geometry, Luigi Bianchi Days Proc. 1st Non-Orthodox School (Warsaw, 21-28 Sep. 1995), ed. D. Wöjcik, J. Cieśliński (Polish Scientific, Warsaw, 1998) 25-36.

[23] C. Rogers and W.K. Schief, Bäcklund and Darboux Transformations, (Camb. Univ. Press, Cambridge, 2002).

[24] D.D. Zhang and D.J. Zhang, Rational solutions to the ABS list: Transformation approach, Symm. Integr. Geom.: Meth. Appl. 13 (2017) No.078 (24 pp). 